\title{
Near Lossless Compression Technique for Bayer Color Filter Images using Wavelets
}

\author{
Lakshmi.M \\ Department of Electronics and Communication \\ Engineering DDCSM Polytechnic \\ College,Palacode-Dharmapuri \\ (Dt),TamilNadu,India-636808.
}

\author{
Allirani.A \\ Department of Electronics and Communication \\ Engineering SRS College of Engineering and \\ Technology, Salem-638316. TamilNadu,India
}

\begin{abstract}
Interpolating full-resolution color images from color-filterarray (CFA) samples is Image demosaicing. Bayer pattern is popular among various CFA patterns and demosaicing with this Bayer pattern produces high resolution color images. This paper presents a new demosaicing approach for spatially sampled image data perceived through a color filter array, and thereby exploiting the correlation of color components for subsampled image reconstruction. The above method is compatible with wavelet-domain denoising before demosaicing. It is also a general framework to apply existing image denoising algorithms to color filter array data. Compression is through Huffman coding and application of biorthogonal wavelets with the results proving that the proposed method is satisfactory in comparison with other techniques available in the literature.
\end{abstract}

\section{General Terms}

Color Filter Array (CFA), Image Demosaicing

\section{Keywords}

Bayer pattern, Biorthogonal wavelets, Huffman coding.

\section{INTRODUCTION}

A minimum of three colour samples at each pixel location is mandatory for production of a colour image. Three fullchannel colour images can be obtained through the use of a colour filter in front of each sensor. Though it requires three charge-coupled device (CCD) sensors it is costly with the sensors having to be aligned correctly [1]. A better costeffective solution will be to place a colour filter array (CFA) before the sensor to capture one colour component in each pixel, interpolating the missing two colour components [2]. Because of CFA's mosaic pattern, such interpolation is called "demosaicing".

Data is obtained through spatial subsampling implemented as a color filter array (CFA), in digital imaging applications, which ensures each pixel location measures on a single colour [3]. The best known of these involves light's primary colours: red, green, and blue with attempts being made to complement human' spatial colour sensitivity through quincunx sampling of green component which has double the density of that of the red and blue. The Bayer pattern CFA [5], with two green, one blue, one red samples' arranged in a $2 \times 2$ square block is extensively used though many CFAs were proposed.

Demosaicing is an inverse reconstruction problem in a spatially under-sampled vector field with primary colour components. The solution to the inverse problem, in the L2 sense of an orthogonal projection on band space functions separately for every spatially subsampled colour channel producing unacceptable visual distortions/artifacts.

An image is a real world sampled version as the original scene's energy is known at discrete positions alone. Sampling generates aliasing if sensor sampling frequency is not high in comparison to the captured scene's maximum frequency. During aliasing, errors are part of the original signal. The Whittaker-Shannon sampling theorem states that when sampling frequency is twice the continuous signal's maximum frequency the original can be recovered as there is no aliasing. The case of CFA images is discussed in [6,7]. A sinc function is used for interpolation in reconstructing the original signal. But this function's support is infinite and decaying and so is not practical. An ideal interpolation function's ideal is often used [8].

Apart from spatial under-sampling in Bayer pattern, this is also deduced to ensure that the observation of values of colour triple exhibit significant correlation specially at high spatial frequencies: such content pointing to the presence of edges, while low-frequency information leads to clearly seen colour content. Demosaicing algorithms in literature try to use spatial frequency domain's correlation structure.

Zhang et al. [9] proposed component decorrelating based on wavelets for efficient lossless colour image compression. An alternative to such wavelet type analysis of CFA images based on the principles of Fourier analysis and filterbanks is conceived. Image denoising based on wavelet-based methods are popular in literature, as shrinkage/thresholding estimators are simple, computationally efficient and also liable to have good theoretical properties, adapting to spatial inhomogeneities well.

This paper proposes a wavelet-based CFA image denoising and demosaicing framework enabling application of existing wavelet-based image denoising techniques on to sparsely sampled data directly. This is important as many noise sources inherent to the charge-coupled device or other imaging technique employed need to be considered. Noise reduction procedures should be put in effect before demosaicing so that interpolation results are improved and also to avoid creating additional correlation structures into noise. Though prior work emphasized demosaicing before denoising, this paper suggests a method which performs wavelet-based denoising and demosaicing together in tandem, naturally.

\section{RELATED WORKS}

Chung et al [10] presented an efficient chroma subsampling strategy to compress DTDI mosaic video sequences in H.264/AVC. Based on color domain transform between RGB domain and YUV domain, a position-selection strategy 
determines both subsampling chroma components, $\mathrm{U}$ and $\mathrm{V}$, according to DTDI mosaic structure. Reconstructed DTDI video sequences quality is better than those reconstructed through conventional methods. Results were superior when experimenting on some DTDI mosaic video sequences. It was better than those used conventional H.264/AVC as compression standard.

Malvar et al [11] presented a low-complexity integerreversible spectral-spatial transform allowing efficient lossless and lossy compression of color filter-array images (referred to as camera-raw images). The new transform's advantage is that it maps pixel array values into a format that is directly compressed in a lossless, lossy, or progressive-to lossless manner by an existing image coder like JPEG 2000 or JPEG $\mathrm{XR}$. Hence no special codec design is needed to compress camera-raw data. Another advantage is the new transform allowing for mild compression of camera-raw data in a nearlossless format and allowing high quality offline postprocessing. But this is with camera-raw files that are half the size of those of existing camera-raw formats.

$\mathrm{Gu}$ et al [12] developed a novel universal demosaicing strategy which would work with any color filter array pattern, based on spatio-spectral sampling theory and a filter bankbased treatment of color image sampling. Under the assumption of high frequency filter bank coefficients sparsity, it was shown that chrominance reconstruction information can be reinterpreted as a robust regression scheme, irrespective of color filter array sampling pattern. Experimental results on multispectral images demonstrated the proposed method's effectiveness.

Condat et al [13] presented a new $2 \times 3$-periodic CFA that provided the optimal trade-off between robustness to aliasing, chrominance noise and luminance noise by construction. Also a simple and efficient linear demosaicing algorithm which exploits CFA's spectral properties is described. Experiments confirmed the new design's superiority both in noiseless and noisy scenarios.

Condat et al [14] proposed a simple demosaicing approach consisting of recovering chrominance by linear filtering and denoising luminance through a classical method for grayscale images. A Matlab implementation of the proposed approach was available online. There are many efficient denoising methods, including the Non-Local Means, for which reported fast implementations. Using an algorithm within the approach opens doors to real-time high-quality demosaicing. The proposed framework can be extended to other CFAs.

\section{METHODOLOGY}

The block diagram of the proposed system is shown in figure 1.

The demosaicing problem of the CFA image may be viewed as the sum of a fully observed green pixel array and sparsely sampled difference images corresponding to red and blue. Specifically, let $n=\left[\begin{array}{ll}n_{0} & n_{1}\end{array}\right]^{T}$ index pixel location and define $x(n)=[r(n) g(n) b(n)]^{T}$ to be the corresponding color triple. If we define difference images $\alpha(n)=r(n)-g(n)$ and $\beta(n)=b(n)$ $-\mathrm{g}(\mathrm{n})$, then the CFA image is given by $\mathrm{y}(\mathrm{n})=\mathrm{g}(\mathrm{n})+\alpha_{\mathrm{s}}(\mathrm{n})+$ $\beta_{\mathrm{s}}(\mathrm{n})$, where

$\alpha_{s}(n)=\left\{\begin{array}{cc}r(n)-g(n) & \text { if } n_{0}, n_{1} \text { even } \\ 0 & \text { otherwise }\end{array}\right.$

$$
\beta_{s}(n)=\left\{\begin{array}{cc}
b(n)-g(n) & \text { if } n_{0}, n_{1} \text { odd } \\
0 & \text { otherwise }
\end{array}\right.
$$

are the sparsely sampled difference images.

The fact that difference channels $\alpha(n)$ and $\beta(n)$ exhibit rapid spectral decay relative to the green channel $g(n)$ follows from the (de-)correlation of color content at high frequencies. Empirically it is seen that the log-magnitude spectra of $g(n)$, $\alpha(n), \beta(n)$, respectively denoted by $G(\omega), A(\omega), B(\omega)$ for frequency variable $\omega=\left[\begin{array}{ll}\omega_{0} & \omega_{1}\end{array}\right]^{T}$.

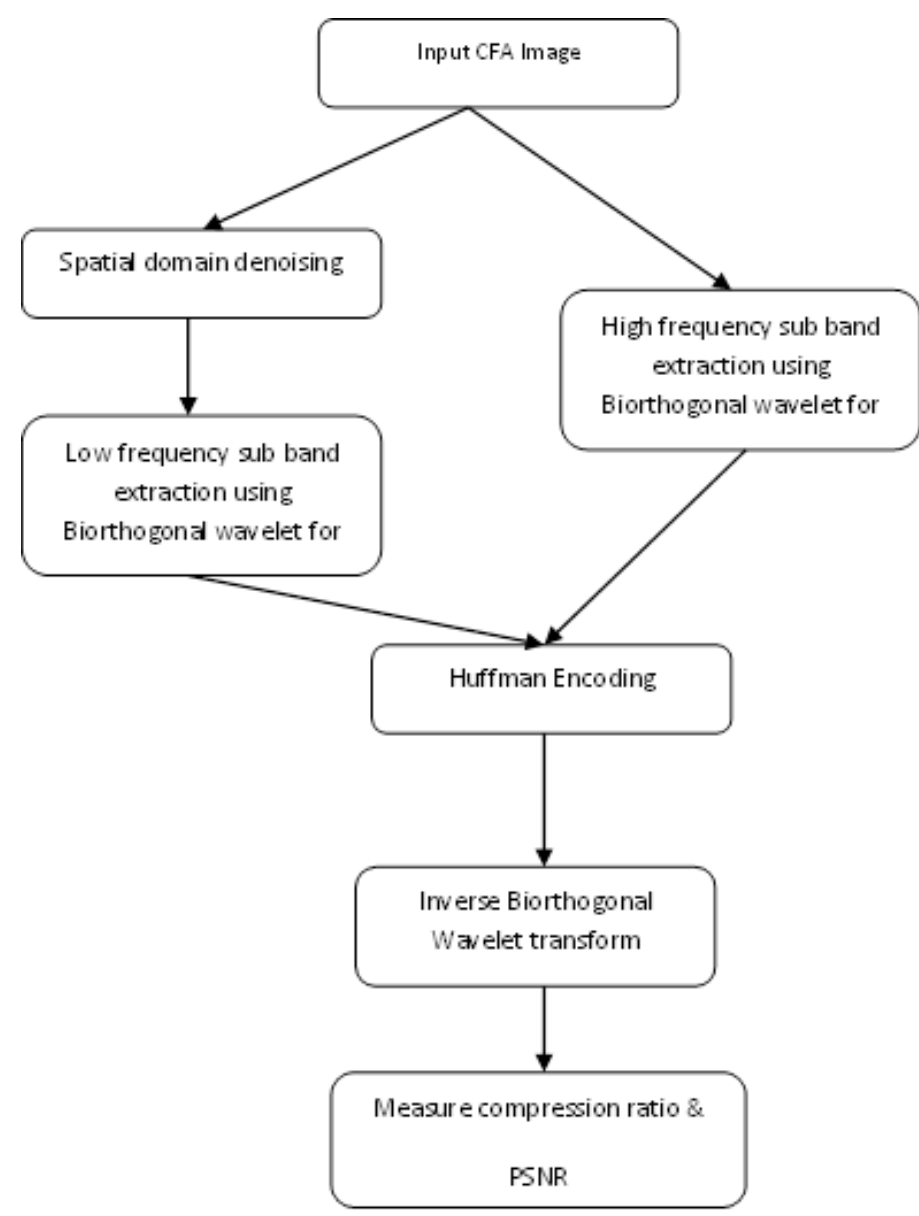

Figure 1: The proposed architecture

The lowpass nature of these subsampled difference signals separate data colour components seen through a colour filter array. A separable wavelet transform equates a convolutions set corresponding to two dimension directional filtering followed by a separable dyadic decimation on spatial frequency axes.

The application of these steps to y(n) results in log-magnitude spectrum after filtering according to the standard directional wavelet filterbank low- and highpass transfer functions $\operatorname{HLL}(\omega), \operatorname{HHL}(\omega), \operatorname{HLH}(\omega)$, and $\operatorname{HHH}(\omega)$, respectively.

Biorthogonal wavelets have compact biorthogonal spline wavelets and compact duals. Analysis and synthesis are assigned to two different functions in biorthogonal wavelets which define separate decomposition and reconstruction filters [15].

The biorthogonal scaling function is given by 


$$
\begin{array}{rr}
\phi(t)=2 \sum_{n=-\infty}^{\infty} h(n) \phi(2 t-n) & \text { dual } \tilde{\phi}(t)=2 \sum_{n=-\infty}^{\infty} \tilde{h}(n) \tilde{\phi}(2 t-n) \\
\langle\phi(t), \tilde{\phi}(t-k)\rangle=\delta(k) \quad\left\langle\phi\left(2^{-k} t\right), \tilde{\phi}\left(2^{-k} t-n\right)\right\rangle=2^{k} \delta(n)
\end{array}
$$

where $h(n)$ and $\tilde{h}(n)$ - impulse response of FIR filters and

$\varphi(t)$ and $\tilde{\varphi}(t)$ - two sets of scaling functions which generate subspaces.

Huffman codes - digital data compression codes - are the result of the work of Prof. David A. Huffman. Huffman encoding is an algorithm for lossless file compression based on occurrence frequency of a symbol in the file under compression [16]. They exploit message entropy and provide good compression ratios. Huffman coding is a statistical coding technique which is the foundation for many compression processes. Such compression techniques include techniques which generate an input data stream duplicate after an expand cycle.

Based on statistical coding the Huffman algorithm is based on symbol probability is directly related to its representation length [17]. The more probable a symbol occurs the sorter its bit-size representation. Files use some characters than others. The number of bits which represent every character is based on the number of characters represented in binary representation. One bit can represent two characters, i.e., 0 is representative of the first character and 1 the second character. So two bits can represent four characters, and so forth. Huffman compression is a variable-length coding system assigning smaller codes for regularly used characters and bigger codes for characters less used unlike ASCII code which has a fixed length and uses 7 bits per character. This process enables reduction of file size to ensure easy transfers.

\section{RESULTS AND DISCUSSION}

In this experiment, the CFA raw data are generated from six standard 24-bit color test images with size $512 \times 512$ which includes 'lena', 'baboon', 'airplane', 'house', and 'peppers'. The interpolated image without compression is regarded as the source image. In the conventional lossless compression method, the interpolated images are compressed by Huffman Coding. The compression ratio (CR) and PSNR of the test images with CFA pattern are shown in Table 1.

Table 1: Compression results for test images

\begin{tabular}{|l|l|l|}
\hline Test Image & Compression Ratio & PSNR \\
\hline Lena & 5.4465 & $41.72 \mathrm{db}$ \\
\hline Airplane & 5.4377 & $38.5 \mathrm{db}$ \\
\hline Peppers & 5.6094 & $36.41 \mathrm{db}$ \\
\hline Baboon & 5.9925 & $31.88 \mathrm{db}$ \\
\hline House & 6.5552 & $37.17 \mathrm{db}$ \\
\hline
\end{tabular}

The average compression ratio achieved by the proposed method is 5.81 which is satisfactory. The average PSNR achieved is $37.14 \mathrm{db}$. The proposed method can provide the high compression ratio and fairly good PSNR. The results prove that the proposed near-lossless compression method is satisfactory.

\section{CONCLUSION}

A lossless image compression for digital image sensors with Bayer CFAs based on a framework for wavelet-based color image analysis and processing was presented. The method is amenable to wavelet-domain denoising before demosaicing, and a general framework to apply existing image denoising algorithms to color filter array data is described. Experiments reveal that this method has higher compression performance but only adequate PSNR than traditional image compression methods and other compression methods.

\section{REFERENCES}

[1] Li, X., Gunturk, B., \& Zhang, L. (2008, January). Image demosaicing: A systematic survey. In Proc. of SPIE (Vol. 6822, pp. 68221J-68221J).

[2] M. Mancuso and S. Battiato. "An Introduction to the Digital Still Camera Technology," ST J. Syst. Res., vol. 2, Dec 2001.

[3] Chung, K. H., \& Chan, Y. H. (2006). Color demosaicing using variance of color differences. Image Processing, IEEE Transactions on, 15(10), 2944-2955.

[4] Rastislav Luckac, and Konstantinos N. Plataniotis, "Color Filter Arrays: Design and performance Analysis", IEEE, transactions on Consumer Electronics, volume 51 No. 4, November 2005.

[5] B.E Bayar, "Color Imaging Arrays," U.S. Patent 3971 065, 1976.

[6] K. Topfer, J.E. Adams, and B.W. Keelan, "Modulation transfer functions and aliasing patterns of CFA interpolation algorithms," Proc. IS\&T PICS, Portland Oregon, 1998, pp. 367-370

[7] J.W. Glotzbach, R.W. Schafer, and K. Illgner, "A method of color filter array interpolation with alias cancellation properties,"Proc. Int'l Conf. on Image Processing, (ICIP'01), Thessaloniki, Greece, vol. 1, Oct. 2001, pp. 141-144.

[8] J.E. Adams Jr., “ Design of practical color filter array interpolation algorithms for digital cameras II," Proc. Int'l Conf. Image Processing, (ICIP'98), Chicago, IL, USA, vol. 1, Oct. 1998, pp. 488-492.

[9] N. Zhang and X. Wu, "Lossless compression of color mosaic images," IEEE Trans. Image Processing, vol. 15, no. 6, pp. 1379-1388, June 2006.

[10] Chung, K. L., Yang, W. J., Chen, C. H., Liao, H. Y. M., \& Zeng, S. M. (2011). Efficient chroma subsampling strategy for compressing digital time delay integration mosaic video sequences in H. 264/AVC. Journal of Electronic Imaging, 20(2), 023011-023011.

[11] Malvar, H. S., \& Sullivan, G. J. (2012, April). Progressive-to-Lossless Compression of Color-FilterArray Images Using Macropixel Spectral-Spatial Transformation. In Data Compression Conference (DCC), 2012 (pp. 3-12). IEEE.

[12] Gu, J., Wolfe, P. J., \& Hirakawa, K. (2010, September). Filterbank-based universal demosaicking. In Image Processing (ICIP), 2010 17th IEEE International Conference on (pp. 1981-1984). IEEE.

[13] Condat, L. (2011). A new color filter array with optimal properties for noiseless and noisy color image acquisition. Image Processing, IEEE Transactions on, 20(8), 2200-2210. 
[14] Condat, L. (2010). A simple, fast and efficient approach to denoisaicking: Joint demosaicking and denoising. vectors, 50,1 .

[15] Goldberg M. A., Pivovarov M. et al., "Application of wavelet compression to digitized radiographs", AJR:163, pp.463-468, 1994.
[16] Singh, S., \& Singh, H. (2012). Improved Adaptive Huffman Compression Algorithm. International Journal of Computers \& Technology, 1(1).

[17] Revankar, P. S., Patil, V. B., \& Gandhare, W. Z. (2010). Data Compression on Embedded System. Information Processing and Management, 535-537. 